\title{
Transfer of directed-forgetting cues across discrimination tasks with pigeons
}

\author{
KAREN L. ROPER and DEVINEY M. CHAPONIS \\ Wake Forest University, Winston-Salem, North Carolina \\ and \\ AARON P. BLAISDELL \\ University of California, Los Angeles, California
}

\begin{abstract}
Directed forgetting is shown as impaired performance on a memory test following an instruction that the presented items will not be tested. Experiments utilizing the delayed matching-to-sample (DMTS) task have demonstrated that this ability to actively control memory is present in animals; however, no study has yet confirmed that cues to forget established in one DMTS discrimination will successfully transfer to other discriminations. Lacking such evidence, it is not clear whether forgetting cues act as "higher level" task instructions or are represented more simply, perhaps as part of a sample-specific sequence of events. The present study revealed good transfer of the forget cue function in pigeons after prior training with the forget cues in a separate discrimination. This finding is discussed in relation to analogous experiments on occasion setting, in which training within more than one discriminative context has been shown to be critical to the transfer of a conditional relation.
\end{abstract}

The need to update our memory is an important cognitive skill, because continued maintenance of unnecessary or out-of-date information would be an intrusive source of interference. It has been shown that people can reduce their recall of targeted information in response to cues indicating that the memory is no longer needed or relevant. A great deal of research has been dedicated to the study of this directed-forgetting ability in humans, primarily with studies that instruct subjects to either remember or forget items from a list. The preponderance of evidence from these investigations shows that people remember better those items that they have been told to remember, as compared with information designated as to be forgotten (see MacLeod, 1998, for a review). Similar effects have been found in analogues of the directed-forgetting paradigm with animals - most typically, pigeons (e.g., Roper, Kaiser, \& Zentall, 1995; see reviews by Grant, 1998; Zentall, Roper, Kaiser, \& Sherburne, 1998), but also rats (Grant, 1982) and monkeys (Roberts, Mazmanian, \& Kraemer, 1984). This research indicates that the ability to actively update and control incoming information is a more general phenomenon and an important skill that can be modeled in a number of species.

Partial funding for this research was provided by a Sigma Xi Grant in Aid of Research awarded to D.M.C. We thank numerous Wake Forest undergraduates for their assistance in running the subjects and, especially, Maggie Sibley for her invaluable help with data entry. We are grateful to Tom Zentall for pointing out that the interaction of the two-way ANOVA rules out a stimulus generalization account of successful F-cue transfer. Correspondence concerning this article should be addressed to K. L. Roper, Department of Psychology, Wake Forest University, WinstonSalem, NC, 27109 (e-mail: roperk@wfu.edu).
Evidence for directed forgetting in animals typically involves delayed matching-to-sample (DMTS) as the underlying memory task. DMTS involves the presentation of a sample object or stimulus (e.g., a hue or line orientation), followed, after a specified delay interval, by a choice between two stimuli, one of which is identical to the preceding sample. A response to the comparison that matches the sample is reinforced. To establish that sample memory may or may not be required for a subsequent matching choice, cues are inserted during the delay that signal whether the trial will end with a choice test. A choice test follows a remember (R-) cue, but not a forget (F-) cue. Directed forgetting is found if a "surprise" choice (e.g., a few interspersed probe trial tests) on trials already signaled with an F-cue results in impaired memory, as compared with performance on equivalent trials involving an R-cue. Unfortunately, although there is ample evidence of directed forgetting in animals with the DMTS procedure, other findings suggest that the phenomenon as studied under these procedures may not be equivalent to comparable tests with humans.

One limitation of the DMTS procedure in assessing the directed-forgetting phenomenon is that the baseline memory task involves the use of the two-choice matching procedure, such as a choice between red and green comparisons after exposure to one of these stimuli in a sample phase. Because the R- and F-cue functions are established within only a limited context, it may be argued that these cues are not functionally equivalent to the more general verbal instructions that are given to humans. Rather than serving as an if-then, or higher level, instruction to forget, delay cues in the typical DMTS procedure may, instead, be represented more simply as part of a sample-specific 
sequence that either does (R-cue) or does not (F-cue) lead to a limited choice of comparisons.

To eliminate this possibility, it would be useful to provide evidence that a forgetting instruction can transfer to stimulus sets other than those involved in training. The one study that has been conducted in this regard (Grant, 1988, Experiments 1 and 2) showed that F-cues for a matching task of one type did not transfer in such a way as to cause forgetting in a matching task of another type. The pigeons in Grant's (1988) first experiment were trained to perform DMTS with two concurrent discrimination tasks, one involving red and green colors as samples and comparisons, and one involving vertical and horizontal lines as the samples and corresponding choice stimuli. R- and F-cues were inserted within the delay interval only on the line discrimination. Probe trial tests revealed the usual directed-forgetting effect on the line discrimination task, but when tests of the F-cue were also introduced into the red/green color task, there was no significant decrease in memory performance. Failure to transfer the effect of the F-cue was evident even when, in Experiment 2, the cue had been trained in each of two discrimination contexts (food/no food and line orientation discriminations) and transferred to a third (color) discrimination. This finding was especially striking since the comparison choice stimuli for each of the discriminations were the same and, thus, should have made use of the same (prospective; see, e.g., Roitblat, 1980; Wasserman, 1986) memory code.

The previous failure to transfer the function of an F-cue to another task setting is reminiscent of the limited transfer effects for features found in early studies of occasion setting. Holland and Lamarre (1984), for example, showed that suppression to Conditioned Stimulus (CS) A (a signal of footshock) could be attenuated when an inhibitory X stimulus (signaling footshock omission) preceded CS A (i.e., $\mathrm{A}+/ \mathrm{X} \rightarrow \mathrm{A}-$ training). (Note: " + " and "-" indicate presence and absence of footshock, respectively, and " $\rightarrow$ " denotes that CS X was presented prior to CS A; events on either side of " " were interspersed within the same treatment session.) Interestingly, the inhibitory properties of Feature X did not transfer to a separately conditioned excitatory CS B. Under these conditions, the ability of X to function as a negative occasion setter was specific only to CS A. In subsequent experiments on occasion setting of Pavlovian conditioning (e.g., Holland, 1986; Rescorla, 1985) and of operant conditioning (Holland, 1991), however, transfer of an occasion setter to other CSs has been successful. The critical determinant, it appears, is whether the conditioned stimuli involved in the transfer task have themselves been targets of occasion setting. For example, the inhibitory effects of negative Feature $\mathrm{X}$ did transfer to $\mathrm{CS} B$ when the subjects had received prior $\mathrm{B}+, \mathrm{Y} \rightarrow \mathrm{B}-$ training (i.e., with Feature $\mathrm{Y}$ as a negative occasion setter for CS B), and the excitatory effects of positive Feature $\mathrm{X}$ transferred to CS B when the subjects had received prior $\mathrm{B}-, \mathrm{Y} \rightarrow \mathrm{B}+$ training (i.e., with Feature $\mathrm{Y}$ as a positive occasion setter for CS B). Holland (1989) argued that a simple associative mechanism may operate when a single CS receives occasion setting training but that a relational mechanism is invoked when multiple CSs receive occasionsetting treatment, each with its own unique feature. The important implication of this is that transfer of the conditional if-then relation may occur only when a conditional rule has been previously established within the targeted context.

The goal of the present study was to determine whether transfer of directed-forgetting cues would occur under conditions in which the opportunity to utilize forget instructions on the transfer stimulus set was explicitly trained from the outset. Pigeons were trained on two sets of R- and F-cues for each of two different DMTS tasks and then were tested for probe trial matching accuracy both following the trained F-cue and on probes with the F-cue transferred from the alternative task.

\section{METHOD}

\section{Subjects}

The subjects were 6 female White Carneaux pigeons (Columba livia) obtained from a breeding colony at the Freidberg campus of Wake Forest University. All the pigeons had served in a previous experiment involving a DMTS task but were naive with respect to the stimuli and contingencies of this experiment. The subjects were maintained at $80 \%$ of their free-feeding weight throughout the experiment. The birds were individually housed in a temperaturecontrolled room lit on a 12:12-h light:dark schedule, with water and grit freely available in their home cages.

\section{Apparatus}

Testing was conducted in two identical $54.5 \times 54.5 \times 54.5 \mathrm{~cm}$ wooden chambers, each located in an isolated room. The birds were placed into the chambers from the front side wall door. Within each box, a touch panel (Carroll Touch, Model 8101-6371-04) was affixed to a $30 \times 24 \mathrm{~cm}$ flat screen monitor (ViewSonic VE150, Model VCLDS21553-1) at one side of the chamber. Affixed to the wall, $11 \mathrm{~cm}$ from the wire-mesh floor and directly across from the touchscreen, was a wireless camera (Model X10) that extended $10 \mathrm{~cm}$ into the box, to allow for video monitoring. Opposite the door, $6 \mathrm{~cm}$ from the floor and $5 \mathrm{~cm}$ from the side wall containing the touch monitor, was a $6 \times 7 \mathrm{~cm}$ opening that allowed access to a feeder magazine delivering mixed grain. Each chamber was interfaced to an IBM NetVista computer in an adjacent room, which ran the experimental programs (generated in Visual Basic programming language), and a $120-\mathrm{V}$ power supply manufactured by Lafayette Instruments to control the feeder mechanism. A white noise generator was used to minimize noise distraction.

\section{Stimuli}

All the stimuli were created in a computer graphics program and were presented against a dark background on the touchscreens. Four different square $(2.5 \times 2.5 \mathrm{~cm})$ stimuli made up the sample and comparison stimuli for the matching task: red (hue, 357; saturation, 100\%), green (hue, 122; saturation, 91\%), blue (hue, 240; saturation, 96\%), and yellow (hue, 65; saturation, 99\%). Samples appeared in the center of the touchscreen, $21 \mathrm{~cm}$ from the bottom of the wire-mesh floor of the operant chamber. The four cues that served as R- and F-cues were also presented in the center location of the screen and consisted of black patterns (dot, cross, square, and diamond) within a white, 2.5 -cm circle. Comparison stimuli, identical in appearance to the samples, appeared $5 \mathrm{~cm}$ to the left and to 
the right of the sample location. The screen was darkened during the intertrial interval (ITI), except for the low level of ambient illumination that was produced by the computer screen.

\section{Procedure}

Because of their previous training, all the pigeons were familiar with the operant chamber and had acquired the touchscreen peck response prior to the start of the present experiment. Thus, training on the matching task with delay interval cues began immediately.

Acquisition of DMTS with delay interval cues. Each trial began with the presentation of one of the four colors of the sample stimuli (shown with equal probability) in the center location of the screen. Following a fixed ratio (FR) 5 pecking requirement to the sample, it was replaced by either an R- or an F-cue for the duration of the delay interval. The stimuli were paired to form two different color-matching tasks, each with an associated pair of delay cues: Red and green samples were followed by the same set of R- and Fcues (square and diamond for half the subjects, dot and cross for the other half) and a choice between red and green comparison stimuli; blue and yellow sample stimuli were followed by the other set of $\mathrm{R}$ - and F-cues (dot and cross for half the subjects, square and diamond for the other half) and a choice of blue and yellow comparison stimuli (see the top panels in Figure 1). On F-cue trials, termination of the cue signaled the end of the delay, and a 10 -sec ITI was initiated without reinforcement. On R-cue trials, the cue terminated following the scheduled delay interval, and a choice between the two comparison stimuli was presented, with left-right position of the correct comparison balanced across trials. Pecks to the incorrect comparison led immediately to the ITI. A single peck to the stimulus that matched the color of the sample was rewarded with 3-sec access to the grain feeder, followed by a 10 -sec ITI. A new trial began after each ITI, and sessions ended when 96 trials (in which each delay cue stimulus appeared equally often) had been completed or when $1.5 \mathrm{~h}$ had elapsed.

The delay interval was initially set at $2 \mathrm{sec}$ and was increased to $4 \mathrm{sec}$ and then $6 \mathrm{sec}$ as each bird reached criterion performance at each delay. Sessions that did not contain 96 trials (i.e., that had timed out) were not considered for evaluation of criterion, which required $87.5 \%$ correct or better on each of the red/green and the blue/yellow color comparison tasks for two consecutive sessions. The pigeons were overtrained on the final, 6-sec delay for an additional five consecutive sessions after reaching criterion and before probe trial tests were inserted.

Forget cue probe sessions. Probe sessions were the same as the delay cue sessions, with the exception that eight additional probe trials were added to each session: Two probes were added within each one-quarter block of trials, with no probe trials scheduled among the first four trials of each session. Four probe trial sequences were arranged, with two F-cue probe tests for each of the red/green and blue/yellow discriminations (i.e., two probe trials per sample type).
Task Type 1

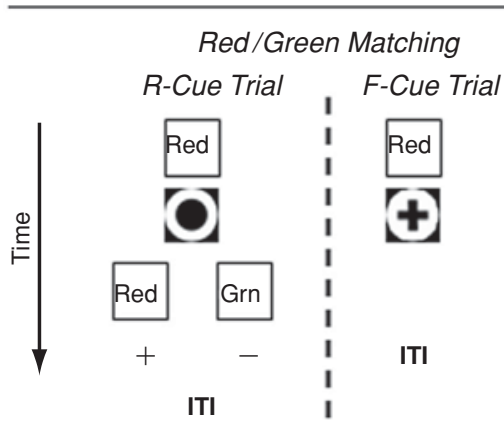

Task Type 1: F-cue Probe Trial Tests

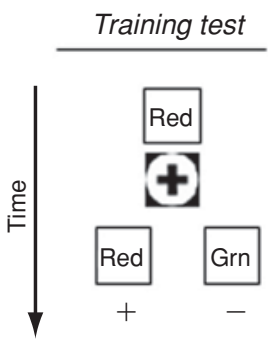

ITI

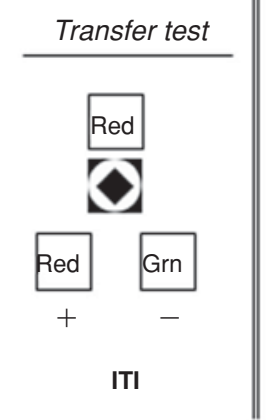

Task Type 2

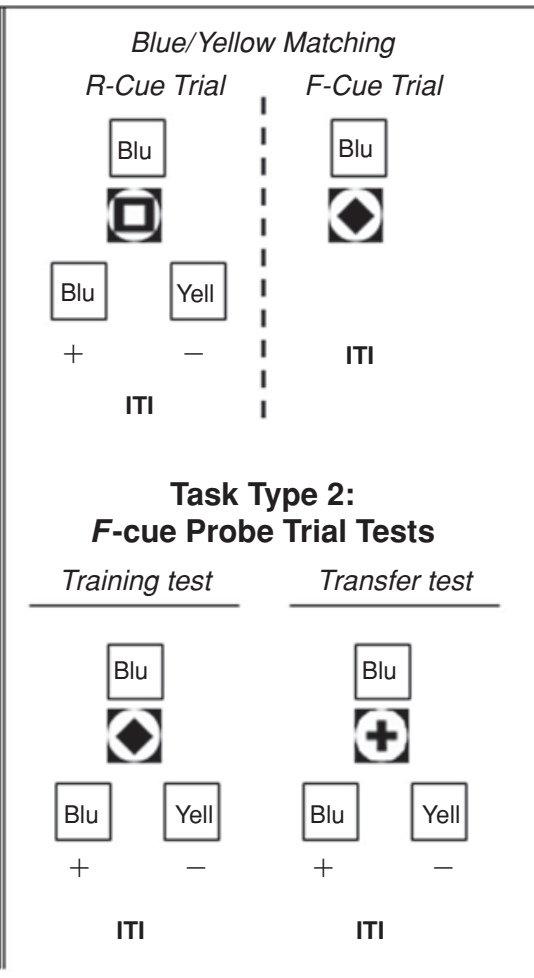

Figure 1. Top: delay cue training procedure with one pair of remember ( $R-)$ and forget $(F-)$ cues for matching to red and green samples (left panel) and another set of $\mathrm{R}$ - and F-cues for matching to blue and yellow samples (right panel). The cue type pairs associated with each color discrimination were reversed for the other half of the pigeons, and the identity of the F-cue within a cue type pair was counterbalanced across subjects. Only those trials with delay interval R-cues ended with a comparison choice test. All trials ended with an intertrial interval followed by the selection of a new trial type. Bottom: F-cue probe trial tests for each matching task, with F-cues that were tested in the trained (left side within each task) or transfer (right side within each task) matching task. 
Each of the probe trials involved the usual FR-5 requirement to a color sample stimulus, followed by an F-cue that filled the 6-sec delay. On these trials, however, the F-cue ended with the presentation of comparison stimuli and reinforcement (or none) contingent upon a peck to the matching comparison stimulus. A 10-sec ITI followed both correct (reinforced) responses and incorrect responses. The position of the correct comparison location was balanced across the eight probe trials.

The pigeons were tested on F-cue probe trials in two phases, each comprising 14 sessions. The test phases were separated by a retraining period without probes (i.e., identical to the original DMTS 6-sec delay acquisition sessions) for 6 consecutive sessions. For half of the birds, the identity of the F-cue during the first phase of probe sessions corresponded to that in the prior training conditions (i.e., F-cues were consistent with the sample color pair that they had been trained with during acquisition of the DMTS task); probe trial comparison tests in the second phase of testing involved samples followed by the F-cue from the transfer task (i.e., the F-cues were those established for the other sample color pair during acquisition of the DMTS task). The order of these probe session phases was reversed for the other half of the pigeons. The design of the experiment is presented in Figure 1.

\section{RESULTS}

\section{Accuracy on the DMTS Task With Delay Interval Cues}

Matching-to-sample performance on R-cue trials with the initial 2 -sec delay was acquired by all the birds in an average of 27.3 sessions, and progression through the 4-sec delay to reach criterion performance at the 6-sec delay was reached within the next 18.2 sessions.

\section{Probe Sessions DMTS Accuracy}

Within each of the two test phases, data were collected across 14 consecutive sessions; however, since some of the birds failed to complete their sessions within the time limit, the data were ultimately pooled across only the first 11 sessions. In the case of incomplete sessions, the data were summarized across the equivalent number of completed R-cue training trials (528 total, consisting equally of red/green and blue/yellow color discrimination trials) and probe trials (44 probes of each color task discrimination).

A four-way mixed ANOVA was conducted on probe trial test data, with test order (training task or transfer task as first probe phase) as the between-subjects factor and task type (red/green or blue/yellow), cue type (R or F), and test type (training or transfer task) as repeated measures. This analysis showed a main effect of test order $[F(1,4)=$ $9.46, p<.05]$, which did not interact with any other single factor or combination of factors, and no effect of task type $[F(1,4)=2.01, p>.20]$. Thus, we pooled across both factors and conducted a two-way repeated measures ANOVA with cue type (R or F) and test type (training or transfer task) as repeated measures. This analysis revealed a main effect of cue type $[F(1,5)=16.71, p<.01]$ but no main effect of test type $(F<1)$. Furthermore, there was an interaction between cue type and test type $[F(1,5)=$ $6.40, p=.05]$.

The main effect of cue type (R vs. F) supports the conclusion that can be drawn from Figure 2 that the F-cues effectively reduced comparison choice accuracy on the color pairs with which they had been trained (training Fcue test) and on the transfer color pairs (transfer F-cue test). Post hoc analyses (Bonferroni test) using the error term from the two-way ANOVA further supported these conclusions. As is shown in Figure 2 (left bars), accuracy was higher on R-cue trials than on F-cue trials across the probe trial test sessions $(p=.01)$. Importantly, this

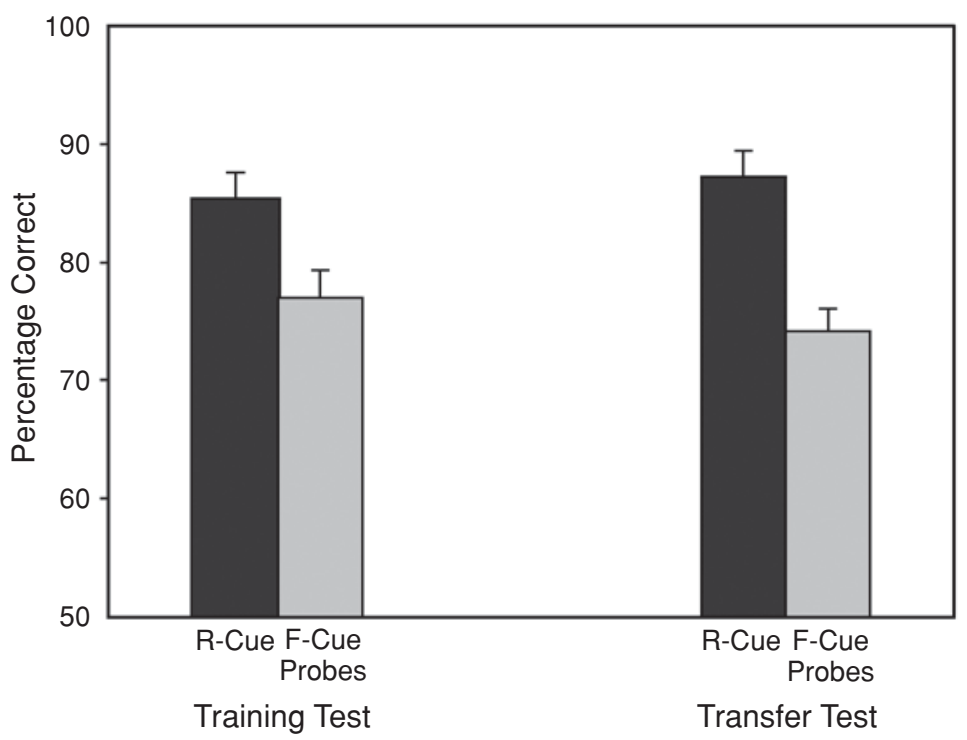

Figure 2. Mean percentages of correct choices (with $S E$ bars) on the delayed matching-to-sample task during probe sessions. At left is remember ( $R$-) cue and forget ( $\mathrm{F}$-) cue probe trial performance when the $\mathrm{F}$-cues were the same as those in training (training test). At right is $\mathrm{R}$-cue and $\mathrm{F}$-cue probe trial performance when the F-cues were transferred from the other color discrimination (transfer test). 
reduction in performance caused by the F-cue training procedure was also apparent when the F-cues came from the transfer color-matching task $(p=.002$; right bars in Figure 2). Accuracy did not differ across tasks for F-cue trials or R-cue trials $(p s>.5)$, indicating that probe trial performance was equivalent despite the change in trial structure. However, although no difference was found across tasks between F- and R-cue probe trials, the interaction revealed by the two-way ANOVA rules out an alternative explanation for the main effect of cue type (discussed below).

\section{DISCUSSION}

The purpose of this research was to evaluate whether providing separate cuing stimuli (R- and F-cues) for each of two different directed-forgetting tasks would enable transfer of the F-cue function across tasks. This was successfully demonstrated using different sets of color stimuli for the matching task (red/green and blue/yellow) and different sets of shape stimuli as the R-and F-cues for each task. This finding suggests that previous failures to obtain evidence that pigeons could transfer an F-cue established in one matching task to a matching task of another type (Grant, 1988, Experiments 1 and 2) might be attributed to the absence of prior training with a forget instruction in the transfer task discrimination.

Although no difference was found across tasks between F- and R-cue probe trials, the interaction revealed by the two-way ANOVA rules out an alternative explanation for the main effect of cue type (raised by a reviewer of an earlier version of this article). There was an insufficient number of birds in our study to achieve counterbalancing across all possible color arrangements (e.g., red, green, blue, and yellow) for the matching tasks. Thus, the two matching tasks used were structured with the same two pairs of colors (red/green and blue/yellow) for all the birds. This raises the logical possibility that there was generalization among the colors in the two pairs. For example, red and yellow may have been coded similarly, and likewise, blue and green may have been coded similarly. Such common coding could promote transfer of F-cue function due to generalization among stimuli, rather than to transfer of F-cue function across tasks.

This scenario also predicts no difference in the effect size observed in the training and the transfer tasks, because they are actually the same task. However, the significant interaction between cue type (R and F) and test type (training and transfer) indicates that the pigeons did perceive a difference between the two types of task, so that the effect of cue type was reliably different for each type of task. This provides evidence that the colors were discriminable and, thus, sample stimulus generalization was not the source of transfer on the F-cue probe tests. What is interesting is that the attenuating effect of the Fcue on matching accuracy is greater in the transfer task. A speculative explanation for the increased effectiveness of the transfer F-cue on test trials is that their unexpected appearance may have increased attention to them. Maki
(1979) found a similar effect in an autoshaping procedure in which pigeons were reinforced in the presence of a vertical, but not a horizontal, stimulus (i.e., vertical $\rightarrow$ food, horizontal $\rightarrow$ no food) in Phase 1, followed in Phase 2 by matching-to-sample training, with a food sample signaling a red stimulus as a correct comparison and a no-food sample signaling a green stimulus as a correct comparison. The pigeons were subsequently tested on combined elements from the two phases with either a consistent mapping (vertical $\rightarrow$ food $\rightarrow$ red/green and horizontal $\rightarrow$ no food $\rightarrow$ red/green) or an inconsistent mapping (vertical $\rightarrow$ no food $\rightarrow$ red/green and horizontal $\rightarrow$ food $\rightarrow$ red/green). Surprisingly, the pigeons performed better on the inconsistent trials than on the consistent trials. Maki suggested that the novel arrangement produced by the inconsistent mapping increased attention to the sample on those trials (relative to consistent trials), which improved accuracy at selecting the correct comparison.

One possible implication of the successful delay interval cue transfer found in our procedure is that, in comparison with the experiments by Grant (1988), our pigeons could not have established a strategy to remember all the sample stimuli from the transfer task discrimination, since F-cues were utilized in that discrimination as well. Roper and Zentall (1993) suggested that the absence of a delay interval cue could come to serve as an implicit R-cue. Grant's pigeons had never been trained to forget the sample stimuli within the transfer task discrimination and, therefore, may have had difficulties abandoning this default remember strategy when F-cue stimuli from the cue-training task were later inserted at test. According to this assessment, transfer across matching tasks of an if-then relation signaled by delay interval cues may, as in the aforementioned studies of occasion setting, be shown to critically depend on explicit prior training with the conditional rule.

Given the importance of the DMTS paradigm to the study of forgetting, this research may be especially important to researchers concerned with the seemingly contextspecific nature of animal memory. For example, White (2001) suggested that performance measures across the different retention intervals within a mixed-delay matching task (involving different lengths of delay on each trial) may be independent of each other. In an important experiment to assess this possibility, White and Cooney (1996) showed that the factors that affect performance even at a particular delay interval (e.g., a change in reinforcer probability, so as to create a specific form of comparison response bias) did not affect performance at other trained delay durations. This implies that the forgetting observed in one discriminative context may be quite specific and, therefore, independent of retention in others. By contrast, the present investigation provides evidence that the effect of a delay interval cue to forget need not be specific to the conditions in which it was formed but that prior experience with the consequences of such cues on an alternative discrimination is important for successful generalization across contexts to occur. This finding offers an important first step in determining how directed-forgetting cues 
function in the DMTS task with animals and may help us to regain the promise of this procedure for making crossspecies comparisons with the human directed-forgetting literature.

\section{REFERENCES}

GRANT, D. S. (1982). Stimulus control of information processing in rat short-term memory. Journal of Experimental Psychology: Animal Behavior Processes, 8, 154-164.

GRANT, D. S. (1988). Directed forgetting in pigeons: Tests of transfer of cue effectiveness across samples from different dimensions. Learning \& Motivation, 19, 122-141.

Grant, D. S. (1998). Directed forgetting in pigeons. In J. M. Golding \& C. M. MacLeod (Eds.), Intentional forgetting: Interdisciplinary approaches (pp. 239-264). Hillsdale, NJ: Erlbaum.

Holland, P. C. (1986). Transfer after serial feature positive discrimination training. Learning \& Motivation, 17, 243-268.

Holland, P. C. (1989). Transfer of negative occasion setting and conditioned inhibition across conditioned and unconditioned stimuli. Journal of Experimental Psychology: Animal Behavior Processes, 15, 311-328.

Holland, P. C. (1991). Acquisition and transfer of occasion setting in operant feature positive and feature negative discriminations. Learning \& Motivation, 22, 366-387.

Holland, P. C., \& Lamarre, J. (1984). Transfer of inhibition after serial and simultaneous feature negative discriminative training. Learning \& Motivation, 15, 219-243.

MACLEOD, C. M. (1998). Directed forgetting. In J. M. Golding \& C. M. MacLeod (Eds.), Intentional forgetting: Interdisciplinary approaches (pp. 1-57). Hillsdale, NJ: Erlbaum.
MAKI, W. S. (1979). Pigeons' short-term memories for surprising vs. expected reinforcement and nonreinforcement. Animal Learning \& Behavior, 7, 31-37.

Rescorla, R. A. (1985). Inhibition and facilitation. In R. R. Miller \& N. E. Spear (Eds.), Information processing in animals: Conditioned inhibition (pp. 299-326). Hillsdale, NJ: Erlbaum.

Roberts, W. A., Mazmanian, D. S., \& Kraemer, P. J. (1984). Directed forgetting in monkeys. Animal Learning \& Behavior, 12, 29-40.

Roitblat, H. L. (1980). Codes and coding processes in pigeon shortterm memory. Animal Learning \& Behavior, 8, 341-351.

Roper, K. L., Kaiser, D. H., \& Zentall, T. R. (1995). True directed forgetting in pigeons may occur only when alternative working memory is required on forget-cue trials. Animal Learning \& Behavior, 23, 280-285.

Roper, K. L., \& Zentall, T. R. (1993). Directed forgetting in animals. Psychological Bulletin, 113, 513-532.

Wasserman, E. A. (1986). Prospection and retrospection as processes of animal short-term memory. In D. F. Kendrick, M. E. Rilling, \& M. R. Denny (Eds.), Theories of animal memory (pp. 53-75). Hillsdale, NJ: Erlbaum.

White, K. G. (2001). Forgetting functions. Animal Learning \& Behavior, 29, 193-207.

White, K. G., \& CoOney, E. B. (1996). Consequences of remembering: Independence of performance at different retention intervals. Journal of Experimental Psychology: Animal Behavior Processes, 22, 51-59.

Zentall, T. R., Roper, K. L., Kaiser, D. H., \& Sherburne, L. (1998) A critical analysis of directed-forgetting research in animals. In J. M. Golding \& C. M. MacLeod (Eds.), Intentional forgetting: Interdisciplinary approaches (pp. 265-287). Hillsdale, NJ: Erlbaum.

(Manuscript received December 13, 2004; revision accepted for publication April 6, 2005. ) 